\title{
COMETA: A Corpus for Medical Entity Linking in the Social Media
}

\author{
Marco Basaldella ${ }^{\dagger, *}$, Fangyu Liu $^{\dagger, *}$, Ehsan Shareghi ${ }^{\dagger, \ddagger}$, Nigel Collier $^{\dagger}$ \\ ${ }^{\dagger}$ Language Technology Lab, University of Cambridge \\ $\ddagger$ University College London \\ $\dagger\{\mathrm{mb} 2313$, fl399, nhc30\}@cam.ac.uk \\ ‡e.shareghi@ucl.ac.uk
}

\begin{abstract}
Whilst there has been growing progress in Entity Linking (EL) for general language, existing datasets fail to address the complex nature of health terminology in layman's language. Meanwhile, there is a growing need for applications that can understand the public's voice in the health domain. To address this we introduce a new corpus called COMETA, consisting of 20k English biomedical entity mentions from Reddit expert-annotated with links to SNOMED CT, a widely-used medical knowledge graph. Our corpus satisfies a combination of desirable properties, from scale and coverage to diversity and quality, that to the best of our knowledge has not been met by any of the existing resources in the field. Through benchmark experiments on 20 EL baselines from string- to neural-based models we shed light on the ability of these systems to perform complex inference on entities and concepts under 2 challenging evaluation scenarios. Our experimental results on COMETA illustrate that no golden bullet exists and even the best mainstream techniques still have a significant performance gap to fill, while the best solution relies on combining different views of data.
\end{abstract}

\section{Introduction}

Social media has become a dominant means for users to share their opinions, emotions and daily experience of life. A large body of work has shown that informal exchanges such as online forums can be leveraged to supplement traditional approaches to a broad range of public health questions such as monitoring suicidal risk and depression (Benton et al., 2017b), domestic abuse (Schrading et al., 2015), cancer (Nzali et al., 2017), and epidemics (Aramaki et al., 2011; Joshi et al., 2019).

One of the widely exercised steps to establish a semantic understanding of social media is En-

\footnotetext{
${ }^{*}$ Equal contribution.
}

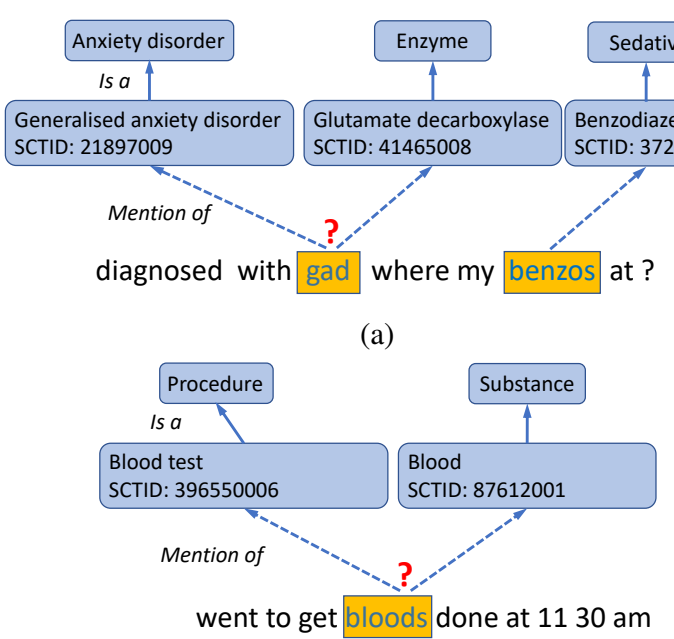

(b)

Figure 1: Examples of the EL inference challenges for user generated text in the health domain.

tity Linking (EL), i.e., the task of linking entities within a text to a suitable concept in a reference Knowledge Graph (KG) (Liu et al., 2013; Yang and Chang, 2015; Yang et al., 2016; Ran et al., 2018). However, it is well-documented that poorly composed contexts, the ubiquitous presence of colloquialisms, shortened forms, typing/spelling mistakes, and out-of-vocabulary words introduce challenges for effective utilisation of social media text (Baldwin et al., 2013; Michel and Neubig, 2018).

These challenges are exacerbated in EL for user generated content (UGC) in the health domain for two main reasons: lack of dedicated annotated resources for training EL models, and entanglement of the aforementioned challenges in general social media with the inherent complexity of the health domain and its terminology (see Table 1).

For example, in Figure 1 we show sentences taken from social media where the semantics of the concept linking is complex and context-dependent. In the first case, "diagnosed with gad where by 


\begin{tabular}{lll}
\hline Input term & Gold SNOMED label & Challenge \\
\hline scratchy throat & Pharyngeal dryness & Colloquial symptom \\
lower right abdomen & Structure of right lower quadrant of abdomen & Term compositionality \\
anti nausea meds & Medicinal product acting as antiemetic agent & Negated term \\
MSM & Dimethul sulfone & Alternative product name \\
up all night cleaning & Obsessive compulsive disorder & Complex inference \\
\hline
\end{tabular}

Table 1: Challenging examples of laymen's terms in COMETA and their target SNOMED concepts.

benzos at", benzos is a colloquial form of benzodiazepines, a type of sedative, and if correctly resolved can provide a contextual clue to assign the appropriate sense to the polysemous term gad: an abbreviation for generalised anxiety disorder rather than e.g. glutamate decarboxylase. In the second example, "went to get bloods done at 11 30", the word bloods could be interpreted literally as blood; however, in this case it clearly refers to a blood test, and it can be correctly resolved only by considering the full context in which it is used.

In this paper we open up a new avenue for EL research specifically targeted at the important domain of health in social media through the release of a new resource: the Corpus of Online Medical EnTities (COMETA), consisting of 20K biomedical entity mentions in English from publicly available and anonymous health discussions on Reddit. Each mention has been been expert-annotated with KG concepts ${ }^{1}$ from SNOMED CT (Donnelly, 2006) ${ }^{2}$, a structured medical vocabulary of ca.350K concepts widely used to code Electronic Health Records (EHRs). As we show, COMETA provides a high quality yet challenging benchmark for developing EL techniques, especially for concepts not encountered during training (zero-shot concepts). Due to its semantic diversity the corpus represents an important pathway to knowledge integration between layman's language, EHRs and research evidence.

Through a set of experiments we shed light on the challenges in this domain for several EL baselines utilising a diverse range of techniques from basic string-matching to low-dimensional entity embeddings (Bojanowski et al., 2017), KG structure embeddings (Grover and Leskovec, 2016; Agarwal et al., 2019), and context aware BERT embeddings (Devlin et al., 2019; Lee et al., 2020). We show

\footnotetext{
${ }^{1}$ Throughout the paper concept refers to nodes in a KG (i.e., SNOMED), term/entity refers to the surface form mention of a concept in text, and context refers to the text in which a term appears. Also, SCTID denotes SNOMED CT Identifier.

${ }^{2}$ We use the July 2019 release of the international edition.
}

a simple augmentation of the mainstream BERT model with a Multi-Level Attention module can improve its effectiveness in capturing the contextual nuances of highly diverse layman's language in the health domain. Our experimental results illustrate that the best solution needs to combine multiple views of data and still heavily relies on basic techniques, while the remaining performance gap highlights the challenging nature of COMETA. We summarise these challenges and underline some of the key areas that are indispensable for further progress in this domain.

\section{Related Work and Datasets}

Entity Linking. EL (Bunescu and Pasca, 2006) is an important task that has sparked attention in recent years due to its wide-scale potential to aid in knowledge acquisition, e.g. the complementary problems of cross-document coreference resolution (Dredze et al., 2016), semantic relatedness (Dor et al., 2018), geo-coding (Gritta et al., 2017) and relation extraction (Koch et al., 2014).

Systems that link entities to Wikipedia (Wikification) (Liu et al., 2013; Roth et al., 2014) and scientific literature to biomedical ontologies (Zheng et al., 2015) have been the focus of attention for many years. Generic EL systems such as Babelfy (Moro et al., 2014) and Tagme (Ferragina and Scaiella, 2011) identify and map entities to Wikipedia and WordNet (Miller et al., 1990) but do not directly integrate the coding standards of healthcare KGs such as SNOMED. Medical EL systems such as cTAKES (Savova et al., 2010) and MetaMap (Aronson and Lang, 2010) were designed to perform medical EL on EHRs but limited evidence e.g. (Denecke, 2014) points to a large drop in recall on UGC such as patient forums.

Medical EL in Social Media. There are several medical EL corpora based on scientific publications (Verspoor et al., 2012; Mohan and Li, 2019), EHRs (Suominen et al., 2013) and death certificates 
(Goeuriot et al., 2017). However, none of these EL corpora dealt with the challenges of UGC.

Due to under-reporting of drug side effects (Freifeld et al., 2014) pharmacovigilance datasets have been among the popular UGC benchmarks for evaluating medical EL. The earliest corpus in this domain was CADEC (Karimi et al., 2015) where 1253 AskAPatient posts (6754 concept mentions) were annotated based on a search for the drugs Diclofenac and Lipitor. Another dataset, Twitter ADR (Nikfarjam et al., 2015), consists of 1784 posts (1280 concept mentions) based on a search for 81 drug names, while TwiMed (Alvaro et al., 2017) provides a comparable corpus of $1 \mathrm{~K}$ PubMed and 1K Twitter texts (3144 concept mentions) based on a search for 30 drugs. Limsopatham and Collier (2016) introduced two Twitter datasets (201 and 1436 concept mentions) with mappings to the SIDER-4 database (Kuhn et al., 2016), and RedMed (Lavertu and Altman, 2019) used Reddit to build a lexicon of alternative spellings for 2978 drugs to improve EL on social media. Closest to our work is MedRed (Scepanovic et al., 2020), a medical Named Entity Recognition corpus of $2 \mathrm{~K}$ Reddit posts based on forums for 18 diseases. However we note several key differences to our work: our corpus is four times larger, provides two levels of mapping to general and context-specific concepts and has a much greater diversity of concepts rather than just symptoms and drugs ( $(3.3)$.

\section{The COMETA Corpus}

The COMETA corpus satisfies multiple properties which we will explain throughout this section:

CONSISTENCY. COMETA has been annotated by biomedical experts to a high quality using SNOMED CT concepts (SCTIDs) - a standard for clinical information interchange ( $\$ 3.2$ );

SCALE AND SCOPE. To the best of our knowledge, with at $20 \mathrm{~K}$ concept mentions, it is the largest UGC corpus for medical EL. Annotated entities cover a wide range of concepts including symptoms, diseases, anatomical expressions, chemicals, genes, devices and procedures across a range of conditions ( $(3.3)$;

Distribution. We release the full corpus along with two sampling strategies (Stratified and Zeroshot) to prevent over-optimistic reporting of performance (Tutubalina et al., 2018): while Stratified is designed to show the ability of systems to recognise known concepts with possibly novel mentions, Zero-shot is designed to test for recognising novel concepts (§3.4).

\subsection{Collection}

In order to build our corpus, we crawled healththemed forums on Reddit using Pushshift (Baumgartner et al., 2020) and Reddit's own APIs. We choose forums satisfying strict constraints, i.e. selecting subreddits where: (i) new content was posted daily, (ii) the quality of the content was sufficient (e.g. avoiding spam-ridden forums), (iii) the focus was the personal experiences or questions of the users. ${ }^{3}$ Applying these criteria, we selected a list of 68 subreddits (see Appendix A.1 for the full list) and crawled all the threads from 2015 to 2018, obtaining a collection of more than $800 \mathrm{~K}$ discussions. This collection was then pruned by removing deleted posts, comments by bots or moderators, and so on.

In order to obtain the candidate entities, we trained the Flair NER system (Akbik et al., 2018) on a corpus of patient discussions from the health forum HealthUnlocked ${ }^{4}$; we then used this system to find medical entities in a random sub-sample of $100 \mathrm{~K}$ discussions of our Reddit set, resulting in over $65 \mathrm{~K}$ distinct named entities being discovered.

Following the standard practices for ethical health research in social media outlined in (Benton et al., 2017a), we then anonymised the corpus to preserve, as far as possible, the privacy of the users. We removed personally identifiable data from messages and we selected terms that were mentioned by at least five users to avoid using terminology particular to a specific user.

Finally, after anonymisation, we hired two professional annotators with Ph.D. qualification in the biomedical domain to annotate the most popular $8 \mathrm{~K}$ tagged entities with SNOMED concepts.

\subsection{Consistency}

The annotation process consisted of two steps:

FirST STEP. We showed the first annotator an entity and up to six random sentences in which it appeared. If the entity was unambiguous, e.g. left

\footnotetext{
${ }^{3}$ For example, acceptable subreddits were $r /$ health, r/cancer, r/mentalhealth, but not $r /$ medical_news/.

${ }^{4}$ The data for this system was provided by HealthUnlocked (https: / / healthunlocked.com/) and cannot be publicly released in compliance with our data access agreement. The usage of this data was approved by the University of Cambridge's School of Humanities and Social Sciences Ethics Committee.
} 
ankle, the annotator had to associate it to the relevant SCTID (e.g. SCTID: 51636004 - Left Ankle) and up to three sentences correctly representing it. Moreover, the first annotator was required to mark NER system mistakes (e.g., wrong type, wrong span, or non-medical entity) to ensure the inclusion of high quality entities. Only $2.1 \%$ of the entities were rejected, confirming the quality of our NER system.

SECOND STEP. The second annotator then tackled the ambiguous entities, selecting up to three possible specific senses, and associating each sense to the relevant examples. This way, we obtained two levels of annotation: The General level, concerned with the literal meaning of the term, and the Specific level, which takes into account the context in which the entity appears.

For example in the sentence "Regarding my eyes, I'm not experiencing cloudiness.", the literal interpretation of the entity cloudiness corresponds to the General SNOMED concept SCTID: 81858005

- Cloudy (qualifier value); however, a contextsensitive assignment which takes into account the word eyes maps the entity to the Specific concept SCTID: 246636008 - Hazy vision. The specific level requires contextual information to be effectively incorporated in the linking step, hence constitutes a more challenging EL task.

The final corpus contains 20015 entities, each are assigned a General and Specific SCTIDs and accompanied by an example sentence from Reddit where the entity is used. We also provide the link to the Reddit thread where the sentence appears (see Appendix A.2 for a sample). Also, contrary to other corpora, we exclude NIL entities, i.e. entities without a corresponding concept in SNOMED.

\subsubsection{Assessing Annotation Quality}

Similar to Mohan and Li (2019), we assessed the quality of the annotation process by asking two pairs of assessors ${ }^{5}$ to assess the quality of $1 \mathrm{~K}$ random annotations (500 per pair of assessors).

Assessor Guidelines. We asked the assessors to evaluate the correctness of the expert assigned concepts on a discrete scale $[1,5], 1$ being completely incorrect, and 5 being completely correct assignments. For example, mapping "chronic back pain" in the sentence "I have chronic low back pain." to

\footnotetext{
${ }^{5} 3$ senior $\mathrm{Ph}$.D. graduates and a PhD candidate in NLP. Note that there was no overlap between Annotators and Assessors.
}

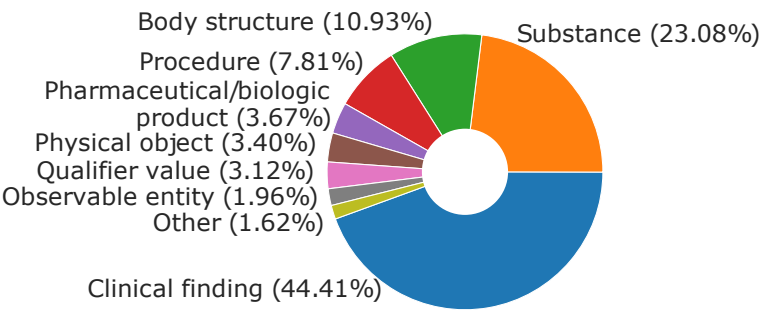

Figure 2: The semantic diversity of SNOMED concepts in COMETA.

SCTID: 134407002 - Chronic back pain entails a score of 5, to SCTID: 61968008 - Syringe entails a score of 1, and to SCTID: 77568009 - Back entails a score of 3 , since the selected node is not correct but it identifies the location of the concept; see Table 8 in the Appendix A. 3 for more details on the instructions we provided to the assessors.

Outcome. Out of $1 \mathrm{~K}$ examples, both assessors assigned the maximum score of 5 to $93.5 \%$ and at least 4 to $96.8 \%$ of both the general and specific level annotations. This is a good indication of the quality of the annotations and is in line with Mohan and Li (2019)'s findings. Further investigation of weakly scored entities (3.2\% of examples) highlights the unique challenges that emerge in this domain. We provide two representative examples: EXAMPLE 1. Regarding the entity " $U I$ " in the sentence "If you're having GI problems, UI issues and/or ED issues please get the breath test for H.Pylori.", the annotator assigned the SCTID: 68566005 - Urinary tract infectious disease. One assessor agreed with the annotator's judgement on considering "UI" as an abbreviation of "Urinary infection", while the other assessor assigned only a score of 3 , considering it as the abbreviation of "Urinary incontinence". Given the sentence, however, both interpretations are plausible.

EXAMPLE 2. Consider the entity "pissed off" in the sentence "And to top it off my stomach becomes bloated and pissed off.". Here, "pissed off" is used figuratively to indicate some form of discomfort; however, the annotator assigned SCTID: 75408008 - Feeling angry which both assessors flagged as incorrect. Nevertheless, both assessors couldn't suggest a better SNOMED concept, as this phrase does not identify a precise disease.

These ambiguities exemplify why performing EL in the UGC domain can be hard even for humans and highlight the complexity found in laymen's medical conversations. 


\subsection{Scale and Scope}

The corpus contains 6404 unique terms, 19911 unique example strings, 3645 unique general concepts (SCTIDs), and 4003 unique specific concepts (SCTIDs). Each general and specific concept is represented on average with more than 1 surface form, while some concepts had more than 15 surface forms, like for example SCTID: 5935008 Oral contraception, SCTID: 225013001 - Feeling bad, and SCTID: 34000006 - Chrohn's Disease.

Additionally, each concept was accompanied by an average of at least 5 example sentences (median of 3), while $4.5 \%$ of entities were linked to different general and specific SNOMED concepts (i.e., due to polysemy or contextual cues). We note that 31 entities are associated to more than one general SCTID, while 453 are associated to more than one specific SCTID.

As illustrated in Figure 2, the most popular SNOMED domains in COMETA are Clinical finding $(44.4 \%)$, Substance $(23.1 \%)$, Body structure (10.9\%), Procedure (7.8\%), and Pharmaceutical / biologic product $(3.7 \%)$, covering more than $90 \%$ of all the entities in the corpus (see Appendix A.4 for more details).

\subsection{Distribution}

We provide the COMETA corpus in two different sampled splits:

STRATIFIED SPLIT. Each SNOMED concept appearing in the test/development sets, appears at least once in the training set. The stratification by SCTID results in $100 \%$ coverage of concepts in test/development, but on the surface form it covers only $58 \%$ of the entities in the test set.

Zero-Shot SPLit. Development and test sets contain only novel concepts for which no training data was available.

In other words, the Stratified split is designed to ensure that the model encounters the same concepts in the training, development and test set, but possibly with different surface forms; the Zero-Shot split, instead, exposes models to unseen terms and concepts in the development and testing sets, making it the hardest of the two settings ( $(4)$. We argue that Zero-Shot is a more realistic setting since obtaining training data that covers all 350K SNOMED concepts involves a very expensive annotation effort. The statistics for the splits are shown in Table 2.

\begin{tabular}{llccc}
\hline & & Training & Dev & Test \\
\hline \multirow{2}{*}{ Stratified } & General & 13489 & 2176 & 4350 \\
& Specific & 13441 & 2205 & 4369 \\
Zero-Shot & General & 14062 & 1958 & 3995 \\
& Specific & 13714 & 2018 & 4283 \\
\hline
\end{tabular}

Table 2: Number of examples in COMETA's splits.

\section{Experiments and Results}

In this section we conduct a diverse set of EL experiments, where we apply different simple and complex paradigms to link the annotated entities (and the sentences in which they appear) with the corresponding SNOMED concepts. We follow previous works in biomedical entity linking and use top- $k$ Accuracy $(k \in\{1,10\})$ to evaluate performance of EL systems (D'Souza and Ng, 2015). Note that Acc@10 is only computed for systems returning a ranked list and measures if the correct concept is contained within the top 10 concepts returned by the system. We also report Mean Reciprocal Rank (MRR, Craswell (2018)), which instead measures the position of the correct concept in the list of concepts returned by the system. Details about training as well as model and hardware configurations are available in Appendix A.5.

Our baselines cover both string/dictionary-based algorithms (§4.1) which are good at capturing surface-level similarities, and neural models capable of incorporating contextual information ( $\$ 4.2)$, where we experiment with a new Multi-Level Attention mechanism based on BERT to allow more efficient incorporation of context. Finally, to achieve the best possible performance, we combine these models in a back-off setting where we leverage the benefits of each paradigm ( $\S 4.3)$. When describing the results, we will report the results on the general split and place the results on the specific split in parentheses.

\subsection{Dictionary and String-based Baselines}

As a first step, we experimented with a set of naïve systems based on string matching and edit distance. ${ }^{6}$ These baselines ignore the context around the entities, since they simply try to match entities against SNOMED labels.

Dictionary. A lookup table is built by traversing the training data, recording every entity and its corresponding SCTID, and directly applied on the test

\footnotetext{
${ }^{6}$ For this set of experiments, we transform all entities and labels to lower-case.
} 


\begin{tabular}{lccc}
\hline & & \multicolumn{2}{c}{ Acc@ 1 } \\
\cline { 3 - 4 }$\#$ & Method & Stratified Split & Zero-Shot Split \\
\hline s.1 & Dictionary & $.51(.45)$ & $0(0)$ \\
\hline s.2 & Exact matching & $.40(.38)$ & $.37(.35)$ \\
s.3 & Levenshtein ratio & $.49(.47)$ & $.52(.49)$ \\
s.4 & Stoilos distance & $.51(.49)$ & $.53(.51)$ \\
\hline s.5 & cTAKES & $.51(.48)$ & $.53(.47)$ \\
s.6 & QuickUMLS & $.31(.30)$ & $.43(.38)$ \\
\hline
\end{tabular}

Table 3: Comparison for Dictionary, String-Matching, cTAKES and QuickUMLS baselines on stratified and zero-shot splits for general and (specific) levels.

set. If an entity is mapped to multiple SNOMED labels, the dictionary records the most frequent one.

String-Matching Edit-Distance. For every term, a string-matching search is conducted on its surface form against all the SNOMED node labels. Note that every SNOMED node has multiple alternative surface forms resulting in 2-36 comparisons per each entity. We count as a hit if the entity is matched with any of the node's surface forms based on exact match, Levenshtein ratio or Stolois distance, two strong string matching heuristics, which are defined as follows: given two strings $x, y$ the Levenshtein ratio (or normalised Levenshtein distance, Yujian and Bo (2007)) is defined as $\frac{\operatorname{Lev}(x, y)}{\max (|x|,|y|)}$ where Lev is the Levenshtein distance (Levenshtein, 1966) between $x$ and $y$; the Stoilos distance (Stoilos et al., 2005) is defined as the similarity of two strings as $\operatorname{comm}(x, y)-\operatorname{diff}(x, y)+$ winkler $(x, y)$ where the first and second terms are commonality and difference scores computed based on lengths of substrings of $x, y$ that are matched/unmatched and the third term is Jaro-Winkler distance (Winkler, 1999). Both edit distance metrics were tuned to offer the best trade-off between true and false positives in the development set; further details are provided in Appendix A.6.

cTAKES. cTAKES (Savova et al., 2010) is a heavily engineered system for processing clinical text. We report on its EL pipeline which is based on several dictionary-based and advanced string matching techniques for resolving abbreviations, acronyms, spelling variants, and synonymy. ${ }^{7}$

QuickUMLS. QuickUMLS (Soldaini and Goharian, 2016) is a fast approximate dictionary

\footnotetext{
${ }^{7}$ We also experimented with feeding the full text (including the entity) to cTAKES, but results were substantially worse.
}

matching system for medical concept extraction using SimString (Okazaki and Tsujii, 2010) as its back-end. We restrict its search space to the SNOMED CT subset of UMLS. As QuickUMLS predicts UMLS CUI instead of SCTID, we map predicted CUIs to SCTIDs through the UMLS api. ${ }^{8}$ When multiple plausible mappings exist, we count a hit if anyone of them matches. ${ }^{9}$

Results. Table 3 summarises the results for the dictionary and string-based baselines. The dictionary method can serve as a strong baseline on the Stratified split, where its performance is barely matched by the more complex string-matching techniques. The most complex strategy, Stoilos distance, outperforms the other string-based techniques, and interestingly is on par with the highly complex cTAKES system while performing significantly better than QuickUMLS. It is worth noting that cTAKES obtained 95.7\% in an EL task on an EHR dataset (Savova et al., 2010), highlighting the greater difficulty of the task when performed on the layman's language typical of UGC.

Additionally, contrary to cTAKES, none of the string-based baselines are relying on external resources which might offer an improvement in resolving some abbreviations or acronyms that our string-based systems miss and cTAKES disambiguates correctly (e.g. "ADHD" to SCTID: 406506008 - Attention deficit hyperactivity disorder). We leave further exploitation of such resources for future work.

\subsection{Neural-based Baselines}

For our neural setting, we define the problem as a cross-space mapping task by representing COMETA entities (along with their contexts) and SNOMED concepts using different text- and graphbased representation learning techniques, and then mapping the learned representations from the textual space to SNOMED concepts space.

Entity Embeddings. We experimented both with "traditional" and contextual embedding techniques. To generate the entity embeddings we use FastText (FT, Bojanowski et al. (2017)) and BioBERT (Lee et al., 2020), a PubMed-specialised version of BERT (Devlin et al., 2019). The former

\footnotetext{
${ }^{8}$ https://documentation.uts.nlm.nih. gov/rest/home.html

${ }^{9}$ Unlike cTAKES, we found that feeding the full text to QuickUMLS yields slightly better results than using the entity only.
} 
was trained and the latter was further specialised on the set of $800 \mathrm{~K}$ Reddit discussions described earlier $(\$ 3.1) .{ }^{10}$ In the case of multi-word terms, their embeddings were generated via averaging. ${ }^{11}$ The dimensionality of the embeddings was 300 for FastText and 768 for BERT, and we denote them as FT-term and BERT-term, respectively. Note that we acknowledge there are alternative options of BioBERT like SciBERT (Beltagy et al., 2019) and ClinicalBERT (Alsentzer et al., 2019). In our own experiments, we discovered that the further specialisation on Reddit discussions is more important than the choice of base model. That said, we leave explorations of other $*$ BERT models on COMETA for future work.

Multi-Level Attention for BERT. As noted by Ethayarajh (2019) the deeper BERT goes, the more "contextualized" its representation becomes. However, interpreting semantics of entities requires contextual knowledge in different degrees and always taking the last layer's output may not be the best solution. In order to address this issue, we propose a Multi-Level Attention (denoted as BERT-term MLA) module on top of BERT to further enhance the representation extracted from BERT by learning how much to attend to each layer for producing an entity representation. The attention weights of the $i$-th layer is computed as $a_{i}=\left[\mathbf{B}_{i} \cdot \mathbf{A}\right]_{+}$, where $[\cdot]_{+}=\max (0, \cdot)$, and $\mathbf{B}_{i} \in \mathbb{R}^{d}$ denotes the representation from the $i$-th level of BERT, $d$ denotes the dimensionality (i.e., here $d=768$ ), and $\mathbf{A} \in \mathbb{R}^{d}$ denotes a trainable attention memory vector. We further normalise $a_{i}$ using a softmax layer, $w_{i} \stackrel{\text { def }}{=} \operatorname{softmax}\left(a_{i}\right)$. Finally, a weighted sum over all layers produces the attention-fused representation, i.e. BERT-term $\mathrm{MLA}_{i}=\sum_{i}^{L} w_{i} \mathbf{B}_{i}$.

Concept Embeddings. We experimented by embedding SNOMED concepts with two modalities: (i) their labels, to exploit textual information, and (ii) their corresponding nodes in the KG, to incorporate the graph structure. Label embeddings were produced by running FastText (denoted as FT-label) and BERT (denoted as BERT-label) on the label,

\footnotetext{
${ }^{10}$ Note that BERT here is used as a feature extractor. We tried finetuning BERT jointly with the alignment model, but performance got worse due to overfitting. We leave properly finetuned BERT models on COMETA as future work.

${ }^{11}$ We tried replacing the entity embeddings with sentence embeddings via RNN/transformers, however, the performance was much worse. We speculate this was due to polluting the informative signal of an entity with its surrounding words. We leave further exploration of this to future work.
}

both trained as described above; for concepts with multiple labels (e.g., SCTID: 61685007 - Lower extremity, Lower limb, Leg), the mean of the label representations is used. For node embeddings, we based our choice of model on the findings reported in Agarwal et al. (2019) and opted for their best reported model for SNOMED, i.e. node2vec (Grover and Leskovec, 2016) with the suggested parameters and vector size $300 .{ }^{12}$

Ensemble Embeddings. We also considered several embeddings that integrate multiple views of the data via (i) concatenation (denoted as $\oplus$ ) of the entity embeddings (e.g, FT-term $\oplus$

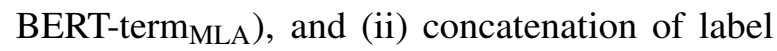
and node2vec embeddings for concepts (e.g., FTlabel $\oplus$ BERT-label $\oplus$ node2vec).

Alignment Model. We adopt a linear transformation followed by ReLU (Nair and Hinton, 2010) for aligning entity and concept embeddings, and we train the model with a max-margin triplet loss:

$$
\mathcal{L}=\sum_{\mathbf{p} \in \mathcal{P}} \max _{\mathbf{t} \in \mathcal{T} \backslash\{\mathbf{t}\}}[\alpha-s(\mathbf{p}, \mathbf{t})+s(\mathbf{p}, \overline{\mathbf{t}})]_{+}
$$

where $\alpha(=0.2)$ is a pre-set margin, $s(\cdot, \cdot)$ is the cosine similarity, $\mathcal{P}$ and $\mathcal{T}$ are the sets of all predictions and target embeddings in a mini-batch, and given a prediction $\mathbf{p}$ and its corresponding ground truth $\mathbf{t}, \overline{\mathbf{t}}$ denotes a negative target embedding.

Results. The results of the neural baselines are presented in Table 4. All individual baselines (n.1 to $n .4)$ fall behind the string-matching methods on Acc@1. This can be due the fact that on average for each entity-concept pair there are less than 4 examples even in the stratified training set, making it difficult for the trained model to generalise well. This issue is more evident in the zero-shot setting.

The ensemble neural baselines compensate for the lack of training signal by leveraging multiple views of the data. As expected, combining both surface and node embeddings of the concepts (n.5) offers a slight improvement, but still fails to match the string-matching baselines. Finally, concatenation of the entity embeddings with our proposed BERT-term ${ }_{\text {MLA }}$ representation, and of the label embeddings with BERT-label (n.6) outperforms all

\footnotetext{
${ }^{12} \mathrm{We}$ also compared node2 vec with more sophisticated model of Kartsaklis et al. (2018) but we observed worse performance. We speculate this is due to the reliance of their model on the presence of textual definitions in SNOMED labels, which is only available in $<4 \%$ of SNOMED nodes.
} 


\begin{tabular}{|c|c|c|c|c|c|c|c|c|}
\hline \multirow[b]{2}{*}{ \# } & \multirow[b]{2}{*}{ term embeddings } & \multirow[b]{2}{*}{ concept embeddings } & \multicolumn{3}{|c|}{ Stratified Split } & \multicolumn{3}{|c|}{ Zero-Shot Split } \\
\hline & & & Acc@1 & Acc@10 & MRR & Acc@1 & Acc@10 & MRR \\
\hline n.1 & FT-term & FT-label & $.40(.38)$ & $.71(.70)$ & $.51(.49)$ & $.21(.20)$ & $.53(.51)$ & $.31(.30)$ \\
\hline n. 2 & FT-term & node2vec & $.17(.12)$ & $.36(.31)$ & $.24(.19)$ & $.01(.03)$ & $.09(.11)$ & $.04(.06)$ \\
\hline n. 3 & BERT-term & BERT-label & $.32(.29)$ & $.58(.56)$ & $.41(.39)$ & $.24(.23)$ & $.50(.50)$ & $.32(.32)$ \\
\hline n. 4 & BERT-term ${ }_{\text {MLA }}$ & BERT-label & $.38(.35)$ & $.66(.63)$ & $.48(.45)$ & $.29(.27)$ & $.56(.52)$ & $.38(.35)$ \\
\hline n. $5^{* *}$ & n.1 & $\mathrm{n} .1 \oplus \mathrm{n} .2$ & $.47(.42)$ & $.76(.73)$ & $.57(.49)$ & $.12(.12)$ & $.37(.41)$ & $.20(.22)$ \\
\hline n. $6^{* *}$ & $\mathrm{n} .1^{*} \oplus \mathrm{n} .4$ & n. $1 \oplus \mathrm{n} .2 \oplus \mathrm{n} .3$ & $.67(.61)$ & $.88(.86)$ & $.74(.70)$ & $.36(.33)$ & $.66(.63)$ & $.46(.43)$ \\
\hline
\end{tabular}

Table 4: Comparison for neural-based baselines on stratified and zero-shot splits for general and (specific) levels.

previous baselines on the stratified split, but still falls behind the string-based baselines on zero-shot.

Compared to Acc@1, while the overall ranking of models remains the same, MRR and Acc@10 are more forgiving. The significant gap between Acc@1 and Acc@10 suggests that a re-ranking step (Liu, 2009) applied to top-10 candidates could further boost the performance. We leave further exploration of this idea to our future work.

\subsection{Back-off Baselines}

To obtain the best possible performance, we experimented with a deterministic back-off procedure (denoted as + ) that applies the Dictionary and backs-off to a String-Matching model $(\S 4.1)$ and finally to the best ensemble model ( $\$ 4.2$; model n.6 in Table 4) for handling the missed cases.

Results. Table 5 reports the Back-off baseline results. The immediate gain on performance compared to each individual counterpart indicates that each model is equipped to tackle only a subset of the underlying challenges in the data. The back-off model combining dictionary, Stoilos distance, and the ensemble neural approach achieves our best performance across both splits (model b. 8 in Table 5). As expected, the neural baselines contribute much less in the Zero-Shot split with a meagre $4 \%(3 \%)$ improvement, compared to the $8 \%(7 \%)$ increase on the Stratified split. Even if their overall contribution is limited, we were able to verify that our neural baselines are actually able to exploit the context as expected. For example w.r.t. the issues typical of the UGC domain we identified in Section 1, we found neural methods helpful in resolving acronyms ("UTIs" to SCTID: 68566005 - Urinary Tract Infection), colloquial synonyms ("bloodwork" to SCTID: 396550006 - Blood Test), compositionality ("drenched in sweat" to SCTID:

\begin{tabular}{|c|c|c|c|}
\hline \multirow[b]{2}{*}{ \# } & \multirow[b]{2}{*}{ Method } & \multicolumn{2}{|c|}{ Acc@1 } \\
\hline & & Stratified Split & Zero-Shot Split \\
\hline b. 1 & s. $1+$ s. 2 & $.66(.59)$ & $.37(.35)$ \\
\hline b. 2 & s. $1+\mathrm{s} .3$ & $.70(.64)$ & $.52(.49)$ \\
\hline b. 3 & s. $1+\mathrm{s} .4$ & $.71(.65)$ & $.53(.51)$ \\
\hline b. 4 & s. $1+$ n. 6 & $.77(.70)$ & $.36(.33)$ \\
\hline b. 5 & s. $2+$ n. 6 & $.71(.67)$ & $.53(.49)$ \\
\hline b. 6 & $\mathrm{~s} .1+\mathrm{s} .2+\mathrm{n} .6$ & $.79(.73)$ & $.53(.49)$ \\
\hline b.7 & s. $1+\mathrm{s} .3+\mathrm{n} .6$ & $.79(.72)$ & $.56(.53)$ \\
\hline b. 8 & s. $1+\mathrm{s} .4+\mathrm{n} .6$ & $.79(.72)$ & $.57(.54)$ \\
\hline
\end{tabular}

Table 5: Back-off baselines on stratified and zero-shot splits for general and (specific) levels.

415690000 - Sweating), complex inference (e.g., "Oral Cancer" to SCTID: 363505006 - Malignant tumour of oral cavity), or even spelling errors combined with alternative product names ("Remicaid" to SCTID: 386891004 - Infliximab, i.e. the active principle of Remicade). This last example is specifically interesting, since the label Remicade is not present in SNOMED but the pre-training of embeddings on medical texts ( $\$ 4.2)$ allowed the neural baselines to pick up the correct node.

\section{Discussion}

The COMETA corpus introduces a challenging scenario for entity linking systems from both ML and NLP perspectives. In this section we summarise these challenges, our findings, and shed light on aspects that demand future attention:

Domain-Specific Language. EL systems similar to our baselines are not uncommon in the biomedical domain: Furrer et al. (2019) used a similar dictionary-BERT ensemble model to achieve the best performance in the 2019 CRAFT Shared Task (Baumgartner et al., 2019) on biomedical literature. However, in their case, the neural component offered a much higher contribution highlighting 
the underlying challenges in medical layman's language. Additionally, probing our proposed MultiLevel Attention for BERT, we observed that a more flexible utilisation of context is effective in understanding the diverse contextual cues.

Low-Resource Regime and Learning. Compared to similar corpora, COMETA has the largest scale. However, from a learning perspective the lack of sufficient regularity in the data could still leave its toll at test phase. This is a natural consequence of high productivity of layman's language in social media, while emerging and unforeseen topics such as pandemics (i.e., COVID19) could also contribute to the problem. In fact, we observed the daunting task that systems face in the zero-shot setting, where in the absence of sufficient training signal, string-based methods offer a strong baseline which is hard to beat for neural counterparts. While we artificially control this in the stratified split we still believe the zero-shot setting draws a more detailed picture of challenges an EL system needs to tackle in a real-world scenario. Further exploration of solutions such as transfer learning across domains (i.e., from medical literature to layman's domain) is beyond the focus of this work, nonetheless COMETA provides the framework for designing and testing such solutions.

Cross-Modality Alignment. While Agarwal et al. (2019) report superior performance of node $2 \mathrm{vec}$ embeddings on several graph-based tasks on SNOMED, this success does not translate into $\mathrm{EL}$ as it relies on mapping across modalities (i.e., text-to-graph). Alternatively, when we replaced the node2vec with concept-label embeddings (produced by FT/BERT) the performance was significantly improved. This suggests that aligning different modalities may require a more complex alignment model or stronger training signals. We leave further exploration of this to future work.

\section{Conclusion}

We presented COMETA, a unique corpus for its scale and coverage which is curated to maintain high quality annotations of medical terms in layman's language on Reddit with concepts from SNOMED knowledge graph. Different evaluation scenarios were designed to compare the performance of conventional dictionary/string-matching techniques against the mainstream neural counterparts and revealed that these models complement each other very well and the best performance is achieved by combining these paradigms. Nonetheless, the missing performance of $28-46 \%$ (depending on the evaluation scenario) encourages future research on this area to take this corpus as a challenging yet reliable evaluation benchmark for further development of models specific to this domain.

COMETA is available by contacting the last author via e-mail or following the instructions on https://www.siphs.org/. We release the pre-trained embeddings and the code to replicate our baselines online at https://github.com/ cambridgeltl/cometa.

\section{Acknowledgments}

Funding: This work was supported by the UK EPSRC (EP/M005089/1). We kindly acknowledge Health Unlocked for making their data available and Molecular Connections Pvt. Ltd ${ }^{13}$ for their work on annotating our data.

\section{References}

Khushbu Agarwal, Tome Eftimov, Raghavendra Addanki, Sutanay Choudhury, Suzanne Tamang, and Robert Rallo. 2019. Snomed2vec: Random walk and Poincaré embeddings of a clinical knowledge base for healthcare analytics. arXiv preprint arXiv:1907.08650.

Alan Akbik, Duncan Blythe, and Roland Vollgraf. 2018. Contextual string embeddings for sequence labeling. In COLING 2018, 27th International Conference on Computational Linguistics, pages 16381649.

Emily Alsentzer, John Murphy, William Boag, WeiHung Weng, Di Jin, Tristan Naumann, and Matthew McDermott. 2019. Publicly available clinical BERT embeddings. In Proceedings of the 2nd Clinical Natural Language Processing Workshop, pages 72 78, Minneapolis, Minnesota, USA. Association for Computational Linguistics.

Nestor Alvaro, Yusuke Miyao, and Nigel Collier. 2017. Twimed: Twitter and pubmed comparable corpus of drugs, diseases, symptoms, and their relations. JMIR public health and surveillance, 3(2):e24.

Eiji Aramaki, Sachiko Maskawa, and Mizuki Morita. 2011. Twitter catches the flu: detecting influenza epidemics using twitter. In Proceedings of the Conference on Empirical Methods in Natural Language Processing (EMNLP), pages 1568-1576.

\footnotetext{
${ }^{13}$ http: //www.molecularconnections.com/
} 
Alan R Aronson and François-Michel Lang. 2010. An overview of metamap: historical perspective and recent advances. Journal of the American Medical Informatics Association, 17(3):229-236.

Timothy Baldwin, Paul Cook, Marco Lui, Andrew MacKinlay, and Li Wang. 2013. How noisy social media text, how diffrnt social media sources? In Proceedings of the Sixth International Joint Conference on Natural Language Processing (IJCNLP), pages 356-364.

Jason Baumgartner, Savvas Zannettou, Brian Keegan, Megan Squire, and Jeremy Blackburn. 2020. The pushshift reddit dataset. arXiv preprint arXiv:2001.08435.

William Baumgartner, Michael Bada, Sampo Pyysalo, Manuel R. Ciosici, Negacy Hailu, Harrison PielkeLombardo, Michael Regan, and Lawrence Hunter. 2019. CRAFT shared tasks 2019 overview - integrated structure, semantics, and coreference. In Proceedings of The 5th Workshop on BioNLP Open Shared Tasks, pages 174-184, Hong Kong, China. Association for Computational Linguistics.

Iz Beltagy, Kyle Lo, and Arman Cohan. 2019. Scibert: A pretrained language model for scientific text. In Proceedings of the 2019 Conference on Empirical Methods in Natural Language Processing and the 9th International Joint Conference on Natural Language Processing (EMNLP-IJCNLP), pages 36063611 .

Adrian Benton, Glen Coppersmith, and Mark Dredze. 2017a. Ethical research protocols for social media health research. In Proceedings of the First ACL Workshop on Ethics in Natural Language Processing, pages 94-102, Valencia, Spain. Association for Computational Linguistics.

Adrian Benton, Margaret Mitchell, and Dirk Hovy. 2017b. Multitask learning for mental health conditions with limited social media data. In Proceedings of the 15th Conference of the European Chapter of the Association for Computational Linguistics (EACL), pages 152-162.

Piotr Bojanowski, Edouard Grave, Armand Joulin, and Tomas Mikolov. 2017. Enriching word vectors with subword information. Transactions of the Association for Computational Linguistics (TACL), 5:135146.

Razvan Bunescu and Marius Pasca. 2006. Using encyclopedic knowledge for named entity disambiguation. In 11th Conference of the European Chapter of the Association for Computational Linguistics (EACL), pages 9-16.

Nick Craswell. 2018. Mean Reciprocal Rank, pages 2217-2217. Springer New York, New York, NY.

Kerstin Denecke. 2014. Extracting medical concepts from medical social media with clinical NLP tools: a qualitative study. In Proceedings of the Fourth
Workshop on Building and Evaluation Resources for Health and Biomedical Text Processing, pages 5460.

Jacob Devlin, Ming-Wei Chang, Kenton Lee, and Kristina Toutanova. 2019. Bert: Pre-training of deep bidirectional transformers for language understanding. In Proceedings of the Conference of the North American Chapter of the Association for Computational Linguistics: Human Language Technologies (NAACL-HLT), pages 4171-4186.

Kevin Donnelly. 2006. SNOMED-CT: The advanced terminology and coding system for eHealth. Studies in health technology and informatics, 121:279.

Liat Ein Dor, Alon Halfon, Yoav Kantor, Ran Levy, Yosi Mass, Ruty Rinott, Eyal Shnarch, and Noam Slonim. 2018. Semantic relatedness of Wikipedia concepts-benchmark data and a working solution. In Proceedings of the Eleventh International Conference on Language Resources and Evaluation (LREC), pages $2571-2575$.

Mark Dredze, Nicholas Andrews, and Jay DeYoung. 2016. Twitter at the grammys: A social media corpus for entity linking and disambiguation. In Proceedings of The Fourth International Workshop on Natural Language Processing for Social Media, pages 20-25.

Jennifer D'Souza and Vincent Ng. 2015. Sieve-based entity linking for the biomedical domain. In Proceedings of the 53rd Annual Meeting of the Association for Computational Linguistics and the 7th International Joint Conference on Natural Language Processing (Volume 2: Short Papers), pages 297302, Beijing, China. Association for Computational Linguistics.

Kawin Ethayarajh. 2019. How contextual are contextualized word representations? comparing the geometry of BERT, ELMo, and GPT- 2 embeddings. In Proceedings of the 2019 Conference on Empirical Methods in Natural Language Processing and the 9th International Joint Conference on Natural Language Processing (EMNLP-IJCNLP), pages 55-65.

Paolo Ferragina and Ugo Scaiella. 2011. Fast and accurate annotation of short texts with Wikipedia pages. IEEE software, 29(1):70-75.

Clark C Freifeld, John S Brownstein, Christopher M Menone, Wenjie Bao, Ross Filice, Taha Kass-Hout, and Nabarun Dasgupta. 2014. Digital drug safety surveillance: monitoring pharmaceutical products in twitter. Drug safety, 37(5):343-350.

Lenz Furrer, Joseph Cornelius, and Fabio Rinaldi. 2019. UZH@CRAFT-ST: a sequence-labeling approach to concept recognition. In Proceedings of The 5th Workshop on BioNLP Open Shared Tasks, pages 185-195, Hong Kong, China. Association for Computational Linguistics. 
Lorraine Goeuriot, Liadh Kelly, Hanna Suominen, Aurélie Névéol, Aude Robert, Evangelos Kanoulas, Rene Spijker, Joao Palotti, and Guido Zuccon. 2017. CLEF 2017 eHealth evaluation lab overview. In International Conference of the Cross-Language Evaluation Forum for European Languages, pages 291303. Springer.

Milan Gritta, Mohammad Taher Pilehvar, Nut Limsopatham, and Nigel Collier. 2017. Vancouver welcomes you! minimalist location metonymy resolution. In Proceedings of the 55th Annual Meeting of the Association for Computational Linguistics (ACL), pages 1248-1259.

Aditya Grover and Jure Leskovec. 2016. node2vec: Scalable feature learning for networks. In Proceedings of the 22nd ACM SIGKDD International Conference on Knowledge Discovery and Data Mining (KDD), pages 855-864.

H Hamacher, H Leberling, and H-J Zimmermann. 1978. Sensitivity analysis in fuzzy linear programming. Fuzzy sets and systems, 1(4):269-281.

Aditya Joshi, Sarvnaz Karimi, Ross Sparks, Cécile Paris, and C. Raina MacIntyre. 2019. Survey of text-based epidemic intelligence: A computational linguistics perspective. ACM Computing Surveys, 52(6):119:1-119:19.

Sarvnaz Karimi, Alejandro Metke-Jimenez, Madonna Kemp, and Chen Wang. 2015. Cadec: A corpus of adverse drug event annotations. Journal of biomedical informatics, 55:73-81.

Dimitri Kartsaklis, Mohammad Taher Pilehvar, and Nigel Collier. 2018. Mapping text to knowledge graph entities using multi-sense LSTMs. In Proceedings of the 2018 Conference on Empirical Methods in Natural Language Processing (EMNLP), pages 1959-1970.

Diederik P Kingma and Jimmy Ba. 2015. Adam: A method for stochastic optimization. In International Conference on Learning Representations (ICLR).

Mitchell Koch, John Gilmer, Stephen Soderland, and Daniel S Weld. 2014. Type-aware distantly supervised relation extraction with linked arguments. In Proceedings of the 2014 Conference on Empirical Methods in Natural Language Processing (EMNLP), pages 1891-1901.

Michael Kuhn, Ivica Letunic, Lars Juhl Jensen, and Peer Bork. 2016. The SIDER database of drugs and side effects. Nucleic acids research, 44(D1):D1075D1079.

Adam Lavertu and Russ B. Altman. 2019. Redmed: Extending drug lexicons for social media applications. Journal of Biomedical Informatics, 99:103307.
Jinhyuk Lee, Wonjin Yoon, Sungdong Kim, Donghyeon Kim, Sunkyu Kim, Chan Ho So, and Jaewoo Kang. 2020. Biobert: a pre-trained biomedical language representation model for biomedical text mining. Bioinformatics, 36(4):1234-1240.

Vladimir I Levenshtein. 1966. Binary codes capable of correcting deletions, insertions, and reversals. In Soviet physics doklady, volume 10, pages 707-710.

Nut Limsopatham and Nigel Collier. 2016. Normalising medical concepts in social media texts by learning semantic representation. In Proceedings of the 54th Annual Meeting of the Association for Computational Linguistics (ACL), pages 1014-1023.

Tie-Yan Liu. 2009. Learning to rank for information retrieval. Foundations and Trends in Information Retrieval, 3(3):225-331.

Xiaohua Liu, Yitong Li, Haocheng Wu, Ming Zhou, Furu Wei, and Yi Lu. 2013. Entity linking for tweets. In Proceedings of the 51st Annual Meeting of the Association for Computational Linguistics (ACL), pages 1304-1311.

Ilya Loshchilov and Frank Hutter. 2019. Decoupled weight decay regularization. In International Conference on Learning Representations (ICLR).

Paul Michel and Graham Neubig. 2018. Mtnt: A testbed for machine translation of noisy text. In Proceedings of the 2018 Conference on Empirical Methods in Natural Language Processing (EMNLP), pages 543-553.

George A Miller, Richard Beckwith, Christiane Fellbaum, Derek Gross, and Katherine J Miller. 1990. Introduction to WordNet: An on-line lexical database. International journal of lexicography, 3(4):235-244.

Sunil Mohan and Donghui Li. 2019. Medmentions: A large biomedical corpus annotated with UMLS concepts. In Automated Knowledge Base Construction $(A K B C)$.

Andrea Moro, Alessandro Raganato, and Roberto Navigli. 2014. Entity linking meets word sense disambiguation: a unified approach. Transactions of the Association for Computational Linguistics (TACL), 2:231-244

Vinod Nair and Geoffrey E Hinton. 2010. Rectified linear units improve restricted boltzmann machines. In Proceedings of the 27th international conference on machine learning (ICML), pages 807-814.

Azadeh Nikfarjam, Abeed Sarker, Karen O'connor, Rachel Ginn, and Graciela Gonzalez. 2015. Pharmacovigilance from social media: mining adverse drug reaction mentions using sequence labeling with word embedding cluster features. Journal of the American Medical Informatics Association, 22(3):671-681. 
Mike Donald Tapi Nzali, Sandra Bringay, Christian Lavergne, Caroline Mollevi, and Thomas Opitz. 2017. What patients can tell us: topic analysis for social media on breast cancer. JMIR medical informatics, 5(3):e23.

Naoaki Okazaki and Jun'ichi Tsujii. 2010. Simple and efficient algorithm for approximate dictionary matching. In Proceedings of the 23rd International Conference on Computational Linguistics (Coling 2010), pages 851-859, Beijing, China.

Chenwei Ran, Wei Shen, and Jianyong Wang. 2018 An attention factor graph model for tweet entity linking. In Proceedings of the 2018 World Wide Web Conference on World Wide Web $(W W W)$, pages 1135-1144. ACM.

Dan Roth, Heng Ji, Ming-Wei Chang, and Taylor Cassidy. 2014. Wikification and beyond: The challenges of entity and concept grounding. In Proceedings of the 52nd Annual Meeting of the Association for Computational Linguistics: Tutorials, page 7.

Guergana K Savova, James J Masanz, Philip V Ogren, Jiaping Zheng, Sunghwan Sohn, Karin C KipperSchuler, and Christopher G Chute. 2010. Mayo clinical text analysis and knowledge extraction system (cTAKES): architecture, component evaluation and applications. Journal of the American Medical Informatics Association, 17(5):507-513.

Sanja Scepanovic, Enrique Martin-Lopez, Daniele Quercia, and Khan Baykaner. 2020. Extracting medical entities from social media. In Proceedings of the ACM Conference on Health, Inference, and Learning, pages 170-181.

Nicolas Schrading, Cecilia Ovesdotter Alm, Ray Ptucha, and Christopher Homan. 2015. An analysis of domestic abuse discourse on Reddit. In Proceedings of the 2015 Conference on Empirical Methods in Natural Language Processing (EMNLP), pages 2577-2583. Association for Computational Linguistics.

Luca Soldaini and Nazli Goharian. 2016. Quickumls: a fast, unsupervised approach for medical concept extraction. In MedIR workshop, sigir, pages 1-4.

Giorgos Stoilos, Giorgos Stamou, and Stefanos Kollias. 2005. A string metric for ontology alignment. In International Semantic Web Conference, pages 624 637. Springer.

Hanna Suominen, Sanna Salanterä, Sumithra Velupillai, Wendy W Chapman, Guergana Savova, Noemie Elhadad, Sameer Pradhan, Brett R South, Danielle L Mowery, Gareth JF Jones, et al. 2013. Overview of the ShARe/CLEF eHealth evaluation lab 2013. In International Conference of the Cross-Language Evaluation Forum for European Languages, pages 212-231. Springer.
Elena Tutubalina, Zulfat Miftahutdinov, Sergey Nikolenko, and Valentin Malykh. 2018. Medical concept normalization in social media posts with recurrent neural networks. Journal of biomedical informatics, 84:93-102.

Karin Verspoor, Kevin Bretonnel Cohen, Arrick Lanfranchi, Colin Warner, Helen L Johnson, Christophe Roeder, Jinho D Choi, Christopher Funk, Yuriy Malenkiy, Miriam Eckert, et al. 2012. A corpus of full-text journal articles is a robust evaluation tool for revealing differences in performance of biomedical natural language processing tools. BMC Bioinformatics, 13:207.

William E Winkler. 1999. The state of record linkage and current research problems. In Statistical Research Division, US Census Bureau. Citeseer.

Yi Yang and Ming-Wei Chang. 2015. S-MART: Novel tree-based structured learning algorithms applied to tweet entity linking. In Proceedings of the 53rd Annual Meeting of the Association for Computational Linguistics and the 7th International Joint Conference on Natural Language Processing (ACLIJCNLP), pages 504-513.

Yi Yang, Ming-Wei Chang, and Jacob Eisenstein. 2016. Toward socially-infused information extraction: Embedding authors, mentions, and entities. In Proceedings of the 2016 Conference on Empirical Methods in Natural Language Processing (EMNLP), pages 1452-1461.

Li Yujian and Liu Bo. 2007. A normalized levenshtein distance metric. IEEE transactions on pattern analysis and machine intelligence, 29(6):1091-1095.

Jin G Zheng, Daniel Howsmon, Boliang Zhang, Juergen Hahn, Deborah McGuinness, James Hendler, and Heng Ji. 2015. Entity linking for biomedical literature. BMC medical informatics and decision making, 15(S1):S4. 


\section{A Appendices}

\section{A.1 Full List of Subreddits}

Table 6 reports the list of 68 subreddits crawled for COMETA.

\section{A.2 Example from COMETA}

Table 7 provides examples from COMETA and illustrates the structure of each line in the corpus.

\section{A.3 Example from Assessor Guidelines}

Table 8 provides an example from the guideline sent to assessors.

\section{A.4 Distribution of Concepts in Stratified and Zero-Shot Splits}

Figure 3 provides the detailed distribution of SNOMED Concepts in Stratified and Zero-Shot splits.

\section{A.5 Reproducibility}

Table 9 and Table 10 describe the hardware and hyperparameters used for the experiments we describe.

\section{A.6 Stoilos Distance}

The commonality function $\operatorname{comm}(x, y)$, is defined as

$$
\operatorname{comm}(x, y)=\frac{2 \cdot \sum_{i} \mid \text { max_common_substring } \mid}{(|x|+|y|) / 2}
$$

Where the max_common_substring between $x, y$ is computed in an iterative manner: first, that of the original $x, y$ are computed; then the common sub-string is removed and search is done again for the next max_common_substring until a threshold of length 3 is met (common sub-strings with $<3$ length are not considered).

The difference function, $\operatorname{diff}(x, y)$, is based on the unmatched part of $x, y$ from the last step. We denote them as $u_{x}, u_{y}$. And the length of them are normalised using a Hamacher product (Hamacher et al., 1978) (a parametric triangular norm):

$$
\operatorname{diff}(x, y)=\frac{\left|u_{x}\right| \cdot\left|u_{y}\right|}{p+(1-p)\left(\left|u_{x}\right|+\left|u_{y}\right|-\left|u_{x}\right| \cdot\left|u_{y}\right|\right)}
$$

We choose $p=0.6$. 


\begin{tabular}{llll}
\hline healthIT & hepc & Cirrhosis & breastcancer \\
AskDocs & T1D & scoliosis & Colic \\
DiagnoseMe & diabetes & health & PsoriaticArthritis \\
cancer & Constipated & cfs & Thritis \\
ChronicPain & Constipation & DuaneSyndrome & fibro \\
dementia & migraine & atrialfibrillation & HiatalHernia \\
flu & panicdisorder & insomnia & PCOS \\
mentalhealth & benzorecovery & DSPD & Urology \\
MultipleSclerosis & Psoriasis & braincancer & multiplemyeloma \\
STD & ClotSurvivors & Hypermobility & leukemia \\
transplant & rheumatoid & GERD & lymphoma \\
birthcontrol & Sciatica & seizures & AskaPharmacist \\
menstruation & urticaria & dialysis & mastcelldisease \\
antidepressants & crazyitch & ChronicIllness & obgyn \\
Allergies & pancreatitis & askdentists & askadentist \\
FoodAllergies & CrohnsDisease & Dentistry & HealthInsurance \\
Allergy & Ovariancancer & Antibiotics & hearing \\
\hline
\end{tabular}

Table 6: The list of the 68 subreddits used as a source for the corpus.

\begin{tabular}{|c|c|c|c|c|c|}
\hline $\begin{array}{l}\text { ID } \\
\text { int }\end{array}$ & $\begin{array}{l}\text { Term } \\
\text { str }\end{array}$ & $\begin{array}{l}\text { General SCTID } \\
\text { int }\end{array}$ & $\begin{array}{l}\text { Specific SCTID } \\
\text { int }\end{array}$ & $\begin{array}{l}\text { Example } \\
\text { str }\end{array}$ & $\begin{array}{l}\text { Subreddit } \\
\text { str }\end{array}$ \\
\hline$\cdots$ & $\cdots$ & $\ldots$ & & & \\
\hline$i$ & acid & 34957004 & 34957004 & I burned myself with acid & AskDocs \\
\hline$i+1$ & acid & 34957004 & 698065002 & acid in my throat & cancer \\
\hline$\ldots$ & $\ldots$ & $\ldots$ & $\ldots$ & $\ldots$ & $\ldots$ \\
\hline
\end{tabular}

Table 7: The structure of the dataset; column names are denoted by bold text, and column types are denoted by monospaced text. The released dataset contains two additional columns, marking the label for the corresponding General and Specific SCTID respectively. However, since a label may appear in multiple nodes, we recommend to always use SCTIDs to retrieve the target nodes.

Please note that the data in this table is used for illustration purposes only and it might not be contained in the released corpus. 


\begin{tabular}{|c|c|c|c|c|}
\hline Quality & Evaluation & Term & Proposed Node & Explanation \\
\hline 5:Excellent & $\begin{array}{l}\text { The SNOMED node } \\
\text { matches exactly the term } \\
\text { or is a synonym of the } \\
\text { term. }\end{array}$ & $\begin{array}{l}\text { Chronic back } \\
\text { pain }\end{array}$ & $\begin{array}{l}\text { Chronic back pain, } \\
134407002\end{array}$ & Exact match. \\
\hline 4:Good & $\begin{array}{l}\text { The SNOMED node is } \\
\text { conceptually similar and } \\
\text { taxonomically close (1-2 } \\
\text { edges) to the target term, } \\
\text { e.g. is a close ances- } \\
\text { tor/descendant or a sib- } \\
\text { ling. }\end{array}$ & $\begin{array}{l}\text { Chronic back } \\
\text { pain }\end{array}$ & $\begin{array}{l}\text { Back pain, } \\
161891005\end{array}$ & $\begin{array}{l}\text { 'Back pain' is the } \\
\text { direct ancestor of } \\
\text { 'Chronic back pain'. }\end{array}$ \\
\hline 3:Fair & $\begin{array}{l}\text { The SNOMED node is } \\
\text { conceptually related and } \\
\text { reasonably close ( } 1 \text { to } \\
3 \text { edges) to the target } \\
\text { term, both taxonomically } \\
\text { or via attributes (finding } \\
\text { site, etc.) }\end{array}$ & $\begin{array}{l}\text { Chronic back } \\
\text { pain }\end{array}$ & Back, 77568009 & $\begin{array}{l}\text { 'back' is the 'finding } \\
\text { site' of 'Chronic back } \\
\text { pain'. }\end{array}$ \\
\hline 2:Poor & $\begin{array}{l}\text { The SNOMED node is } \\
\text { conceptually distant } \\
\text { from the term, and there } \\
\text { is a reasonably long ( } 3-4 \\
\text { edges) path from it to the } \\
\text { correct node }\end{array}$ & $\begin{array}{l}\text { Chronic back } \\
\text { pain }\end{array}$ & Torso, 22943007 & $\begin{array}{l}\text { 'Chronic Back Pain' is } \\
\text { located in the 'Torso', } \\
\text { so they are somewhat } \\
\text { related, and the two } \\
\text { nodes are not far (dis- } \\
\text { tance 3) }\end{array}$ \\
\hline 1:Very Poor & $\begin{array}{l}\text { The SNOMED node is } \\
\text { completely unrelated } \\
\text { with the term, and the path } \\
\text { between the correct node } \\
\text { and the target one is very } \\
\text { long }(>5) \text {. }\end{array}$ & $\begin{array}{l}\text { Chronic back } \\
\text { pain }\end{array}$ & Syringe, 61968008 & $\begin{array}{l}\text { 'Chronic Back Pain' } \\
\text { and 'Syringe' have } \\
\text { high distance (5), } \\
\text { and the concepts are } \\
\text { completely unrelated. }\end{array}$ \\
\hline
\end{tabular}

Table 8: An example from assessor guidelines. 


\begin{tabular}{lr}
\hline hardware & specification \\
\hline RAM & 64 GB \\
CPU & AMD $^{\circledR}$ Ryzen 9 3900x 12-core 24-thread \\
GPU & NVIDIA $^{\circledR}$ GeForce RTX 2080 Ti $(11$ GB $) \times 2$ \\
\hline
\end{tabular}

Table 9: Hardware specifications of the machine used to run our experiments.

\begin{tabular}{lr}
\hline hyper-parameters & search space \\
\hline optimiser & $\left\{\right.$ AdamW $W^{*}$, Adam $\}$ \\
learning rate & $\left\{1 e-4^{*}, 5 e-4,1 e-5^{\dagger}\right\}$ \\
batch size & $\left\{64^{*}, 128,256\right\}$ \\
training epochs & $\left\{30,50^{*}, 100\right\}$ \\
$\alpha$ in Eq. (1) & $\left\{0.05,0.1,0.2^{*}\right\}$ \\
threshold for Levenshtein (b.7) & {$[0.10,0.20]$} \\
threshold for Stoilos (b.8) & {$[0.05,0.10]$} \\
BERT pre-training global step & $\left\{10 \mathrm{k}, 100 \mathrm{k}^{*}\right\}$ \\
BERT pre-training max_seq_length & $\left\{64^{*}, 128\right\}$ \\
\hline
\end{tabular}

Table 10: This table lists the search space for hyper-parameters; ${ }^{*}$ denotes the ones used to obtain the performance described in this publication if not specified otherwise. ${ }^{\dagger}$ identifies parameters used only for models n.5 and n.6. More details can be found in the source code available online at redacted. Details of the two optimisers are specified in Loshchilov and Hutter (2019) and Kingma and Ba (2015).
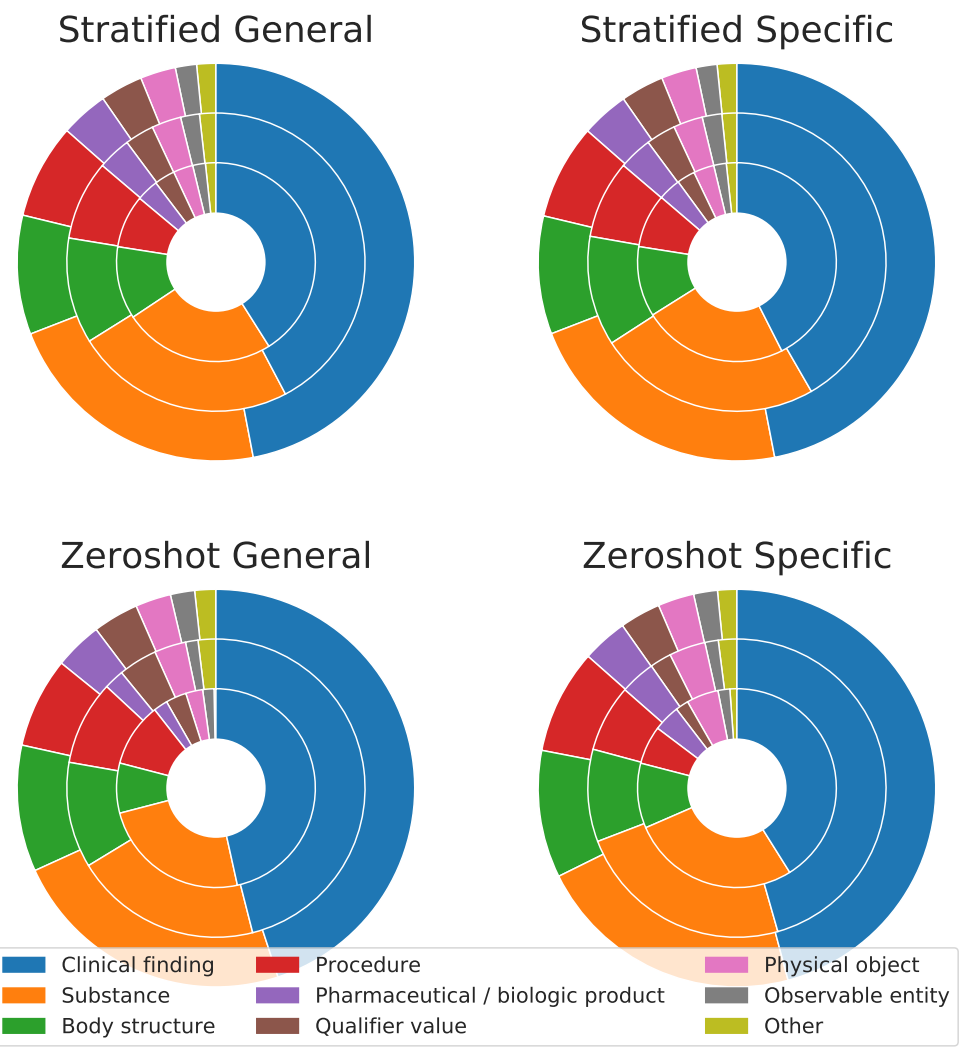

Figure 3: The categories in the dataset by split. The outer pie is the training set, the middle pie is the test set, the inner pie is the development set. 